Discrete Comput Geom 34:637-657 (2005)

DOI: $10.1007 / \mathrm{s} 00454-005-1189-8$

\title{
Drapeability* $^{*}$
}

\author{
John M. Maki, ${ }^{1}$ John E. Wetzel,${ }^{1}$ and Wacharin Wichiramala ${ }^{2}$ \\ ${ }^{1}$ University of Illinois at Urbana-Champaign, \\ Urbana, IL 61801, USA \\ johnmaki@math.uiuc.edu \\ j-wetzel@uiuc.edu \\ ${ }^{2}$ Faculty of Science, Chulalongkorn University, \\ Bangkok 10330, Thailand \\ wacharin@sc.chula.ac.th
}

\begin{abstract}
An arc in the plane is drapeable if it lies in the convex hull of a convex arc of the same length. We establish some fundamental properties of drapeability, and we investigate some sufficient conditions for an arc to be drapeable.
\end{abstract}

\section{Introduction}

A set in the plane is a cover for a family of planar arcs if it contains a congruent copy of each arc in the family. Problems of finding small convex covers, sometimes of prescribed shape, for a given family of planar arcs have been called "worm" problems in the literature. Few such problems have been solved.

Nearly 40 years ago L. Moser [15] (see also [16]) asked for the smallest cover for the family of all arcs of unit length in the plane. The smallest area for a convex cover is known to lie between 0.21946 and 0.27381 [17, p. 417], but little more is known. For an overview of the current status of this problem, see [26].

The convex cover of least area for the smaller family of all convex arcs of unit length is also of considerable interest. Johnson et al. [12] describe such a cover of area less than 0.24656 , and Wichiramala [22] has recently found one with area 0.24640 .

In 1963 Graham [11] asked for the shortest arc that does not fit in an open equilateral triangular region of side one. Besicovitch [1] soon described an arc of length $\sqrt{27 / 28} \approx$ 0.98198 that does not fit, and he conjectured that it is the shortest such arc. The equivalent worm problem, to show that the smallest equilateral triangular cover for the family of all arcs of length one has side $\sqrt{28 / 27}$, remains unresolved.

* Wacharin Wichiramala was supported in part by Grants for Development of New Faculty Staff from Chulalongkorn University. 
Equally unsettled is a conjecture of Wetzel's in the 1970s that a $30^{\circ}$ sector of radius one, whose area is $\pi / 12 \approx 0.26180$, is a cover for the family $\mathcal{F}$ of all planar arcs of unit length. Coulton [3] has recently shown that this sector is a cover for all convex unit arcs, but the details have not been published.

For an overview of such problems, see, for example, [24]. Finch [8] includes a survey of some related questions, and Finch and Wetzel [9] consider an interesting, closely related question.

When investigating covering problems for arcs of unit length, one quickly realizes that convex arcs are generally more easily dealt with than non-convex arcs. Unit arcs that lie in the convex hull of a convex unit arc, arcs that we call drapeable, are, of course, automatically included when their convex "drapes" are accommodated. In this article we investigate some of the fundamental properties of drapeability in the hope of better understanding what it means for a unit arc not to be drapeable, i.e., not to lie in the convex hull of any convex arc of the same or lesser length. To this end we establish a variety of sufficient conditions for an arc to be drapeable. Drapeability has more to do with the "global extent" of the arc than with its local properties, and it is clear that a drapeable arc can be arbitrarily complicated in the small. Useful necessary and sufficient conditions for drapeability seem completely beyond reach.

\section{Drapeable Arcs}

We work always in the coordinate plane $\mathbb{R}^{2}$. Arcs are continuous, rectifiable, and parameterized on the closed interval [0,1]. An arc $\gamma$ is oriented from its initial point $P=\gamma(0)$ to its final point $Q=\gamma(1)$. We write $l(\gamma)$ for its length, $d$ for the distance $P Q$ between its endpoints, and $\{\gamma\}=\{\gamma(t): 0 \leq t \leq 1\}$ for its range, which we call its trace. We write $\mathcal{F}$ for the family of all arcs of length one. Much of what we say applies equally to arcs whose length is at most one; we call such an arc a unit arc.

Definitions. The convex hull of an arc $\gamma$ is the closed convex hull of its trace $\{\gamma\}$. An $\operatorname{arc} \gamma$ is convex if it is simple (i.e., one-one except that its endpoints may coincide) and it lies on the boundary of its convex hull $\mathcal{H}$ (Fig. 1(a)). A convex $\operatorname{arc} \delta$ with distinct endpoints $P$ and $Q$ is a drape if it lies in a closed semi-infinite strip bounded by the endpoint line segment $P Q$ and the rays perpendicular to $P Q$ at the endpoints $P$ and $Q$ (Fig. 1(b)). An arc $\gamma$ is drapeable if it lies in the convex hull of some drape $\delta$ with $l(\delta) \leq l(\gamma)($ Fig. 1(c) ).

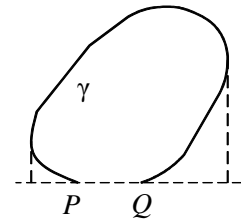

(a) A convex arc

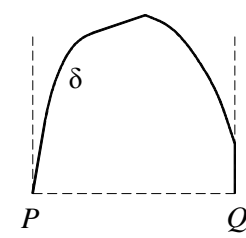

(b) A drape

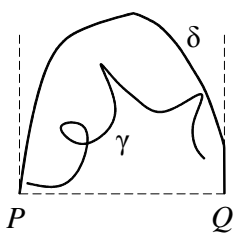

(c) A drapeable arc.

Fig. 1. The basic definitions. 
It is virtually obvious that every convex arc is drapeable (see Fig. 1(a)). Indeed, an arc is drapeable if and only if it lies in the convex hull of some convex arc of the same length. Two non-drapeable Z-shaped unit arcs are pictured in Fig. 6, below.

We close this preliminary section with the useful observation that if the boundary of the convex hull of an arc contains a segment longer than its endpoint segment, then the arc is drapeable.

Theorem 1. If the boundary curve $\partial \mathcal{H}$ of the convex hull $\mathcal{H}$ of an arc $\gamma$ with endpoints $P, Q$ and length $L$ has a line segment $X Y$ with $X Y \geq P Q$, then $\gamma$ is drapeable.

Proof. Recall that the boundary of the convex hull of a closed arc has length at most the length of the arc (see, for example, [20]). Suppose $\gamma$ has length $L$, and let $\gamma^{\prime}$ be the closed arc of length $L+d$ formed by closing $\gamma$ with its endpoint line segment. The boundary $\partial \mathcal{H}$ has the form $\mu \oplus X Y$ where $\mu$ is a convex arc, and it follows that

$$
X Y+l(\mu)=l(\partial \mathcal{H}) \leq l\left(\gamma^{\prime}\right)=L+P Q .
$$

So $l(\mu) \leq L+P Q-X Y \leq L$, and $\mu$ is a suitable drape for $\gamma$.

Corollary 2. Every closed arc is drapeable, as is every arc that lies entirely in one of the closed half-planes whose edge is the line through its endpoints.

\section{Z-Shaped Arcs}

Three-segment polygonal unit arcs are of especial interest, in part because of the longstanding conjecture that for a convex region to contain a congruent copy of every unit arc it is sufficient for it to contain a congruent copy of each such arc [10].

Definition. A three-segment polygonal arc $\zeta=A B C D$ is Z-shaped (Fig. 2) if (a) the convex hull of its vertex set $\{A, B, C, D\}$ is a quadrilateral, (b) the endpoint segment $A D$ meets the middle segment $B C$, and (c) $\max \{B D, C A\}<A D$.

Note that the vertices of a Z-shaped arc are not collinear.

Our first observation is that a non-drapeable three-segment polygonal arc must be Z-shaped.

Lemma 3. Every three-segment polygonal arc that is not Z-shaped is drapeable.

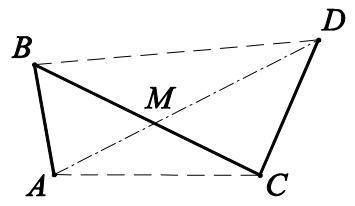

Fig. 2. A Z-shaped arc. 
Proof. Let $\zeta=A B C D$ be a three-segment polygonal arc that is not drapeable. The convex hull of the vertex set $\{A, B, C, D\}$ of $\zeta$ is a line segment, a triangle, or a convex quadrilateral. In the first case $\zeta$ is trivially drapeable, and in the second its drapeability follows immediately from Theorem 1; so the convex hull of $\zeta$ must be a convex quadrilateral. The vertices of $\zeta$ are in one of three possible cyclic orders on the perimeter of that quadrilateral: $A-B-C-D, A-B-D-C$, and $A-C-B-D$. If $A-B-C-D$, then $\zeta$ is convex and consequently drapeable; and if $A-C-B-D$, then the polygonal path $A C B D$ is a convex arc shorter than $\zeta$ that surrounds $\zeta$. So $A-B-D-C$ (Fig. 2), and the segments $A D$ and $B C$ meet because $B$ and $C$ are on opposite sides of $A D$. Finally, if $B D \geq A D$ or if $C A \geq A D$, then $\zeta$ is drapeable by Theorem 1 , and it follows that $\max \{A C, B D\}<A D$. Thus $\zeta$ must be Z-shaped.

\section{Centrally Symmetric Z-Shaped Arcs}

A general necessary and sufficient condition for an arbitrary Z-shaped arc to be drapeable seems difficult to find, but the centrally symmetric case is straightforward. In this section we establish a necessary and sufficient condition for a centrally symmetric Z-shaped arc to be drapeable. We suppose throughout the remainder of this section that the given Z-shaped arc has unit length.

The Shortest Drapes. Let $\mathcal{Z}$ denote the family of all centrally symmetric Z-shaped $\operatorname{arcs} \zeta=A B C D$ of length one. For $\zeta=A B C D$ in $\mathcal{Z}$ the quadrilateral $A B D C$ is a parallelogram (Fig. 3). Let $M$ be the common midpoint of $A D$ and $B C$, write $a=A B=$ $C D$, so that $B M=M C=\frac{1}{2}-a$, and let $e=A M=M D$, so that $A D=d=2 e$. Let $s=B D=A C$. Finally, let $x$ be the width of the infinite strip bounded by the lines $A B$ and $C D$ and let $y$ be the width of the strip bounded by the lines $A C$ and $B D$. Our first objective is to list all of the minimal drapes that surround $\zeta$ and determine which one is the shortest.

The details of the configuration depend on whether the angle $\alpha=\angle C A B$ is acute or obtuse. Let $X, X^{\prime}, Y$, and $Y^{\prime}$ be the feet of the perpendiculars as pictured in Fig. 3, and

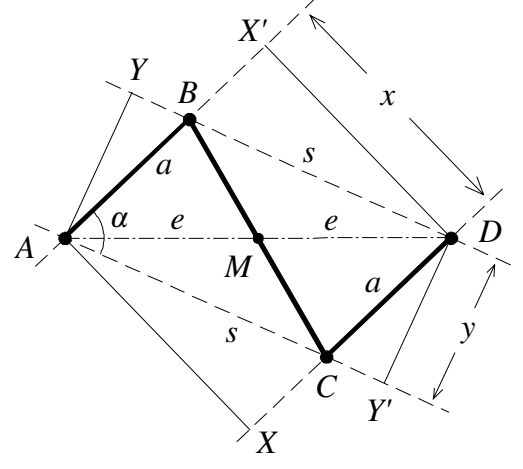

(a) $\alpha<90^{\circ}$.

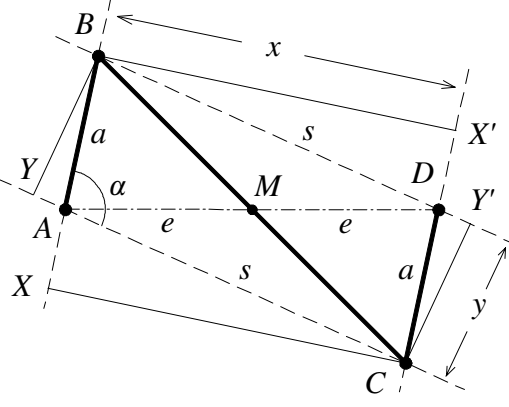

(b) $\alpha \geq 90^{\circ}$.

Fig. 3. Centrally symmetric Z-shaped arcs. 
let

$$
u= \begin{cases}A Y^{\prime}=D Y & \text { when } \quad \alpha<90^{\circ} \\ B Y^{\prime}=C Y & \text { when } \quad \alpha \geq 90^{\circ}\end{cases}
$$

and

$$
v= \begin{cases}A X^{\prime}=D X & \text { when } \quad \alpha<90^{\circ}, \\ B X=C X^{\prime} & \text { when } \quad \alpha \geq 90^{\circ} .\end{cases}
$$

The eight minimal drapes that surround $\zeta$ are listed with their lengths in Table 1. They occur in congruent pairs, with $\delta_{i}^{\prime} \cong \delta_{i}$ in the notation of the table. The shortest drape about $\zeta$ is the shortest of these eight minimal drapes, and $\zeta$ is drapeable if and only if the length of that shortest drape is not larger than one.

We need to determine which of these minimal drapes is the shortest, i.e., which of the lengths $u, v, x, y$ is the smallest. Observe first that $x \leq u$ and $y \leq v$. (Indeed, $x=A X<A C<A Y^{\prime}=u$ when $\alpha<90^{\circ}$, and $x=B X^{\prime} \leq B D \leq B Y^{\prime}=u$ when $\alpha \geq 90^{\circ}$; and precisely similar arguments show that $y \leq v$.) So irrespective of the size of $\alpha$, we have

$$
\begin{aligned}
& l\left(\delta_{2}\right)=l\left(\delta_{2}^{\prime}\right) \geq l\left(\delta_{3}\right)=l\left(\delta_{3}^{\prime}\right), \\
& l\left(\delta_{4}\right)=l\left(\delta_{4}^{\prime}\right) \geq l\left(\delta_{1}\right)=l\left(\delta_{1}^{\prime}\right) .
\end{aligned}
$$

Consequently the minimal drapes $\delta_{2}, \delta_{2}^{\prime}, \delta_{4}$, and $\delta_{4}^{\prime}$ need not be considered further. It is worth mentioning that the size of $\alpha$ plays no further role in these considerations.

To compare $x$ and $y$, we note that

$$
\begin{aligned}
& x=s \sin \alpha, \\
& y=a \sin \alpha .
\end{aligned}
$$

Hence $x / y=s / a$, and it follows that $x \lesseqgtr y$ precisely when $a \gtreqless s$. Consequently the shortest drape is $\delta_{1}$ when $a \leq s$ and $\delta_{3}$ when $a \geq s$.

A Necessary and Sufficient Condition. The results of the previous section suggest using $a$ and $s$ to parameterize $\mathcal{Z}$, so that triangle $A B C$ has sides $A B=a, B C=1-2 a$, and $C A=s$. We call the pair $(a, s)$ the index of $\zeta$ and write $\zeta=\zeta_{(a, s)}$. It follows from

Table 1. The minimal drapes and their lengths.

\begin{tabular}{ccc}
\hline Drape $\left(\alpha<90^{\circ}\right)$ & Length & Drape $\left(\alpha \geq 90^{\circ}\right)$ \\
\hline$\delta_{1}=A B D Y^{\prime}$ & $a+s+y$ & $\delta_{1}=Y B D C$ \\
$\delta_{1}^{\prime}=Y A C D$ & $a+s+y$ & $\delta_{1}^{\prime}=B A C Y^{\prime}$ \\
$\delta_{2}=A C D Y$ & $a+s+u$ & $\delta_{2}=B D C Y$ \\
$\delta_{2}^{\prime}=D B A Y^{\prime}$ & $a+s+u$ & $\delta_{2}^{\prime}=C A B Y^{\prime}$ \\
$\delta_{3}=A C D X^{\prime}$ & $a+s+x$ & $\delta_{3}=B D C X$ \\
$\delta_{3}^{\prime}=X A B D$ & $a+s+x$ & $\delta_{3}^{\prime}=C A B X^{\prime}$ \\
$\delta_{4}=A B D X$ & $a+s+v$ & $\delta_{4}=B A C X^{\prime}$ \\
$\delta_{4}^{\prime}=X^{\prime} A C D$ & $a+s+v$ & $\delta_{4}^{\prime}=C D B X$ \\
\hline
\end{tabular}




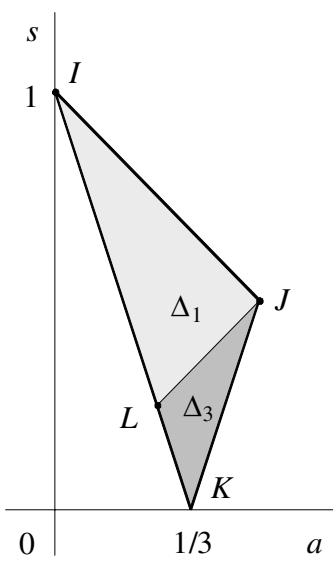

(a) The two regions.

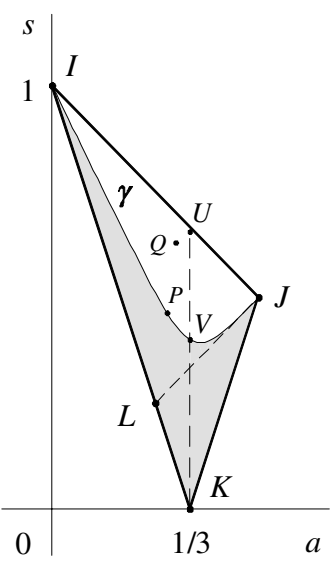

(b) The drapeable region.

Fig. 4. The basic regions.

Stewart's theorem (see p. 6 of [4]) that the distance $d$ between the endpoints of $\zeta_{(a, s)}$ is given by

$$
d^{2}=2 s^{2}-2 a^{2}+4 a-1 .
$$

As $\zeta_{(a, s)}$ ranges over $\mathcal{Z}$, its index $(a, s)$ fills the interior of a triangle $I J K$ in the $(a, s)$ plane whose sides are determined by the triangle inequalities $1-a>s$ and $s>|3 a-1|$ and whose vertices are the points $I(0,1), J\left(\frac{1}{2}, \frac{1}{2}\right)$, and $K\left(\frac{1}{3}, 0\right)$ (Fig. 4(a)).

The line $s=a$ meets triangle $I J K$ at $L\left(\frac{1}{4}, \frac{1}{4}\right)$ and also at $J$, dividing the interior of $I J K$ into two open triangular regions $\Delta_{1}=I L J$, where $s \geq a$ (and the shortest drape is $\delta_{1}$ ), and $\Delta_{3}=K J L$, where $s \leq a$ (and the shortest drape is $\delta_{3}$ ).

The area $(A B C)$ of triangle $A B C$ is given by Heron's formula $(A B C)=\frac{1}{4} \sqrt{\sigma(a, s)}$, where

$$
\sigma(a, s)=\left((1-a)^{2}-s^{2}\right)\left(s^{2}-(1-3 a)^{2}\right),
$$

and also by $(A B C)=\frac{1}{2}$ as $\sin \alpha$, and hence

$$
\sin \alpha=\frac{1}{2 a s} \sqrt{\sigma(a, s)} .
$$

Consequently, from (1) and (2) we see that

$$
x=s \sin \alpha=\frac{1}{2 a} \sqrt{\sigma(a, s)}
$$

and

$$
y=a \sin \alpha=\frac{1}{2 s} \sqrt{\sigma(a, s)},
$$

and it follows that the shortest drape about $\zeta(a, s)$ has length

$$
\left\{\begin{array}{l}
L_{1}(a, s)=l\left(\delta_{1}\right)=a+s+\frac{1}{2 s} \sqrt{\sigma(a, s)} \quad \text { when } \quad(a, s) \in \Delta_{1}, \\
L_{3}(a, s)=l\left(\delta_{3}\right)=a+s+\frac{1}{2 a} \sqrt{\sigma(a, s)} \quad \text { when } \quad(a, s) \in \Delta_{3} .
\end{array}\right.
$$


Let $s=\gamma(a)$ be the level curve $L_{1}(a, s)=1$ in triangle $I J K$ (drawn in Fig. 4(b)). One can verify that $\gamma$ decreases from $I(0,1)$ to a minimum point $\left(a_{0}, s_{0}\right)$ (numerical investigations show that $a_{0} \approx 0.358476$ and $s_{0} \approx 0.392550$ ) and then rises to $J\left(\frac{1}{2}, \frac{1}{2}\right)$, remaining above the line $s=a$. Isolating the radical, squaring, and removing the factor $x+y-1$ shows that $\gamma$ is also given implicitly as the level curve $\mathcal{P}(a, s)=0$ in $I J K$ of the cubic polynomial

$$
\mathcal{P}(a, s)=9 a^{3}-9 a^{2} s+3 a s^{2}+5 s^{3}-15 a^{2}+6 a s-3 s^{2}+7 a-s-1 .
$$

To summarize: $l\left(\delta_{1}\right)>,=,<1$ precisely when $\mathcal{P}(a, s)>,=,<0$, when $s>,=$, $<\gamma(a)$, and, finally, when $(a, s)$ lies above, on, or below $\gamma$ in the interior of triangle $I J K$. It follows that $\zeta_{(a, s)}$ is drapeable if and only if $\mathcal{P}(a, s) \leq 0$.

Dealing ad hoc with the cases in which the vertices are collinear, we find, finally, a necessary and sufficient condition for a centrally symmetric three-segment polygonal arc to be drapeable:

Theorem 4. Let $\zeta=A B C D$ be a centrally symmetric three-segment polygonal arc of length one, and let $a=A B$ and $s=A C$. Then $\zeta$ is drapeable if and only if

(a) $s=1-a$, so that the vertices of $\zeta$ are collinear in the order $A-B-C-D$ and $(a, s)$ lies on the line segment IJ in Fig. 4(b), or

(b) $9 a^{3}-9 a^{2} s+3 a s^{2}+5 s^{3}-15 a^{2}+6 a s-3 s^{2}+7 a-s-1 \leq 0$, so that (a,s) lies in the shaded region in Fig. 4(b).

Corollary 5. If $s \leq s_{0}$, then the $\operatorname{arc} \zeta_{(a, s)}$ is drapeable.

Corollary 6. For each $\varepsilon>0$ there is a non-drapeable Z-shaped arc of length one such that $1>d>s>1-\varepsilon$.

One could, of course, express the conditions of the theorem in terms of other parameterizations of $\mathcal{Z}$, obtained by parameterizing triangle $A B C$ in a different manner. In particular, one could obtain conditions in terms of $a$ and $d$, or, perhaps, in terms of $a$ and $\angle A B C$. The resulting formulas seem equally unpleasant.

\section{Some Special Z-Shaped Arcs}

We note here a few special cases. The familiar Besicovitch $Z$ (Fig. 5), a centrally symmetric three-segment polygonal arc described by Besicovitch [1] in connection with Graham's covering problem, has length $\sqrt{27 / 28}$ (found by Steven Knox in 1994). Scaled

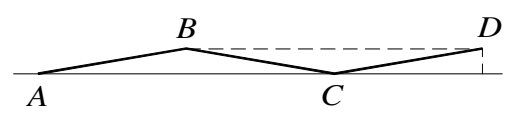

Fig. 5. The Besicovitch $z$. 


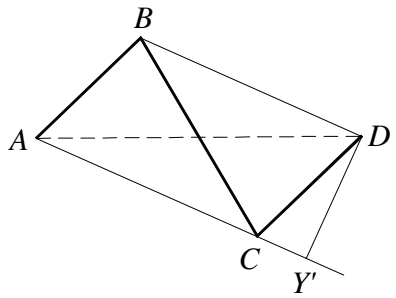

(a) The $\operatorname{arc} \zeta_{1}$.

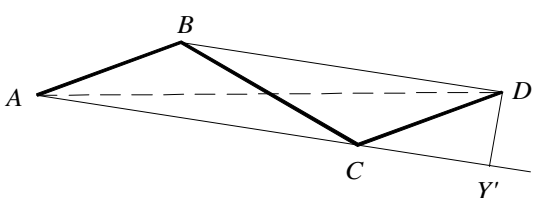

(b) The $\operatorname{arc} \zeta_{2}$.

Fig. 6. Two special Z-shaped unit arcs.

to unit length, it lies in the family $\mathcal{Z}$. Its index is $\left(\frac{1}{3}, \sqrt{\frac{3}{7}}\right)$, the point $U$ in Fig. 4(b), and it is not drapeable. More generally, the index $(a, s)$ of an $\operatorname{arc} \zeta=A B C D$ in $\mathcal{Z}$ for which $A B=B C=C D=\frac{1}{3}$ lies on the line segment $a=\frac{1}{3}, 0 \leq s \leq \frac{2}{3}$ (Fig. 4(b)); and it follows from Theorem 4 that $\zeta$ is drapeable if and only if $s \leq \frac{2}{5}$, or, equivalently, if and only if $A D \leq \sqrt{97} / 15 \approx 0.65659$.

Next we describe two specific Z-shaped arcs of length one for future reference. In each case the given indices were found "experimentally" using Geometer's Sketchpad, and refined numerically. Then the values of $a$ and $s$ were fixed as the six-decimal numbers given, and the resulting data were computed.

The arc $\zeta_{1}=A B C D$ (Fig. 6(a)) with index $(0.278194,0.463300)$ (the point $P$ in Fig. 4(b)) is not drapeable-indeed, calculations show that its minimum width is $t=D Y^{\prime} \approx 0.2585066$, so that its shortest drape $A B D Y^{\prime}$ has length $A B+B D+D Y^{\prime} \gtrsim$ $1.0000006>1$. Its endpoint separation is $d=A D \approx 0.622323$. This arc is interesting because its endpoints are relatively close together for a non-drapeable arc and because its minimum width, $D Y^{\prime} \approx 0.258507$, is relatively large.

The $\operatorname{arc} \zeta_{2}=A B C D$ (Fig. 6(b)) with index $(0.299286,0.629194)$ (the point $Q$ in Fig. 4(b)) also is not drapeable. Its minimum width is $t=D Y^{\prime} \approx 0.152137$, and its shortest drape $A B D Y^{\prime}$ has length about 1.080617 . Its endpoint separation is $d=A D \approx$ 0.899872 . This arc is interesting because the length of its shortest drape is relatively large.

We refer to both these special Z-shaped unit arcs in the next section.

\section{Some Interesting Constants}

In this section we define some constants that are relevant to drapeability, and we give some crude estimates for each. Recalling that $\mathcal{F}$ is the family of all arcs of length one, let $d_{\gamma}, D_{\gamma}$, and $t_{\gamma}$ be the endpoint separation, the diameter, and the minimum width (thickness) of the $\operatorname{arc} \gamma$ in $\mathcal{F}$. The constants we consider are

$$
\begin{aligned}
& K_{1}=\operatorname{lub}\left\{r: \text { every arc } \gamma \in \mathcal{F} \text { with } d_{\gamma} \leq r \text { is drapeable }\right\}, \\
& K_{2}=\operatorname{lub}\left\{r: \text { every arc } \gamma \in \mathcal{F} \text { with } D_{\gamma} \leq r \text { is drapeable }\right\}, \\
& K_{3}=\operatorname{glb}\left\{r: \text { every arc } \gamma \in \mathcal{F} \text { with } t_{\gamma} \geq r \text { is drapeable }\right\}, \\
& K_{4}=\operatorname{glb}\{r: \text { every arc in } \mathcal{F} \text { has a drape of length } r\} .
\end{aligned}
$$


The Endpoint-Separation Constant $K_{1}$. If the endpoints of $\gamma$ are close together, one would expect $\gamma$ to be drapeable, but by Corollary 6 there are non-drapeable arcs with large endpoint separation. The best bounds we know for $K_{1}$ are

$$
0.2221<K_{1}<0.6223 .
$$

The non-drapeable Z-shaped arc $\zeta_{1}$ described above gives the upper bound. The lower bound is the assertion of the following:

Theorem 7. An arc $\gamma$ of length one and endpoint separation $d$ with

$$
d \leq \frac{\pi-2}{\pi+2} \approx 0.2221
$$

is drapeable.

Proof. The minimum width $w$ of a closed arc of length $K$ is at most $K / \pi$ (indeed, the rectangle with side $K / \pi$ and diagonal $K$ is a cover for the family of all closed curves of length $K$; see [21]). Let $\gamma^{\prime}$ be the closed arc of length $L=1+d$ formed by closing $\gamma$ with its endpoint segment $P Q$. Let $R=A B C D$ be a rectangle of width

$$
A D=B C \leq \frac{1+d}{\pi}
$$

circumscribed about $\left\{\gamma^{\prime}\right\}$, with contact points $X$ on $A D, Y$ on $B C, U$ on $D C$, and $V$ on $A B$ (Fig. 7). The points $X$ and $Y$ divide the boundary $\partial \mathcal{H}$ of the convex hull of $\gamma^{\prime}$ into two complementary convex arcs $\gamma_{u}$ and $\gamma_{v}$, labeled so that $\gamma_{u}$ passes through $U$ and $\gamma_{v}$ passes through $V$. Then the drapes

$$
\begin{aligned}
& \delta_{1}=A X \oplus \gamma_{u} \oplus Y B, \\
& \delta_{2}=D X \oplus \gamma_{v} \oplus Y C
\end{aligned}
$$

both surround $\gamma$; and the shorter one, call it $\delta$, has length

$$
\begin{aligned}
l(\delta) & \leq \frac{1}{2}\left(l\left(\delta_{1}\right)+l\left(\delta_{2}\right)\right) \\
& \leq \frac{1}{2}(A X+D X+l(\partial \mathcal{H})+Y B+Y C)
\end{aligned}
$$

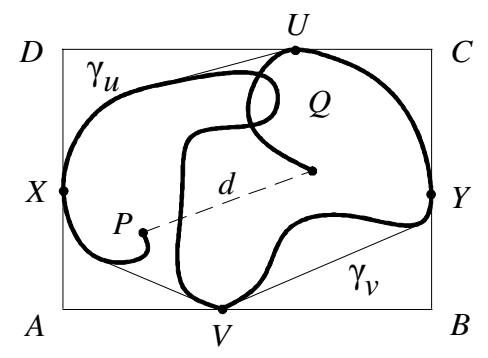

Fig. 7. The two drapes. 


$$
\begin{aligned}
& \leq A D+\frac{1}{2} l\left(\gamma^{\prime}\right) \\
& \leq \frac{1+d}{\pi}+\frac{1}{2}(1+d)=(1+d) \frac{\pi+2}{2 \pi} .
\end{aligned}
$$

However, $(1+d)(\pi+2) / 2 \pi \leq 1$ precisely when $d \leq(\pi-2) /(\pi+2)$.

The Diameter Constant $K_{2}$. If the diameter of an arc $\gamma$ of length one is small, then the arc should have small "global extent" and consequently should be drapeable; but there are $\mathrm{Z}$-shaped arcs of diameter near one that are not drapeable. The diameter $A D \approx 0.6223$ of the non-drapeable Z-shaped arc $\zeta_{1}$ provides an upper bound for $K_{2}$. To find a weak lower bound, recall the well-known theorem of Pál [18] that the regular hexagon of minimal width $D$ (Fig. 8), whose edges have length $D / \sqrt{3}$, is a "universal cover" for sets of diameter $D$. (See also Section D15 of [5].) Then each arc of length one whose diameter satisfies the inequality $D \leq \frac{1}{2}(\sqrt{3}-1) \approx 0.36603$ lies in a regular hexagon of side $D / \sqrt{3}$ and consequently within a drape of length $(1+\sqrt{3}) D \leq 1$ (Fig. 8). Consequently for $K_{2}$ we have the estimate

$$
0.3660 \leq K_{2} \leq 0.6223 .
$$

By replacing Pál's hexagon by a universal cover that has a shorter drape (see [5]), one could raise the lower bound a little.

The Thickness Constant $K_{3}$. If the minimum width $t$ of an $\operatorname{arc}$ in $\mathcal{F}$ is large, one would expect the arc to be more or less rotund and consequently drapeable. An upper bound for $K_{3}$ is provided by the thickness $b_{0} \approx 0.43893$ of the broadworm, the unique $\operatorname{arc}$ in $\mathcal{F}$ whose thickness is as large as possible (see, for example, [24] and the references cited there). A lower bound is the thickness 0.25851 of the non-drapeable arc $\zeta_{1}$ described above. So we have the bounds

$$
0.2585<K_{3}<0.4389 .
$$

The Drapeability Constant $K_{4}$. The length 1.0806 of the shortest drape that contains $\zeta_{2}$, the non-drapeable Z-shaped arc described above, provides a lower bound. Every arc of length one lies in a semicircle of length $\pi / 2 \approx 1.571$, because a semidisk of radius $\frac{1}{2}$ is a cover for $\mathcal{F}$ (see [23]); but we can do a little better. Using lines parallel and perpendicular to the endpoint line $P Q$ (or to any support line in case the arc is closed), encage the arc in an $\ell \times w$ rectangle (Fig. 9). Reflect the endpoints $P$ and $Q$ and contiguous arcs as shown. Then $P^{*} Q^{*} \leq 1$ (because they are joined by a unit arc), and it follows from the

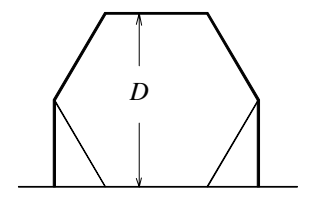

Fig. 8. A universal drape. 


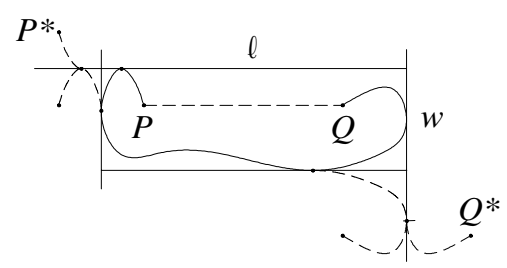

Fig. 9. Geometry of the upper bound.

inequality of the means that

$$
\ell+2 w \leq 2 \sqrt{\frac{\ell^{2}+(2 w)^{2}}{2}} \leq \frac{2}{\sqrt{2}} P^{*} Q^{*} \leq \sqrt{2}
$$

(cf. p. 727 of [14]). A similar argument can be given for each order in which the points of contact of the arc with the edges of the rectangle appear along the arc. So a drape formed by the sides of the rectangle has length $\ell+2 w \leq \sqrt{2}$. It follows that

$$
1.0806 \leq K_{4}<\sqrt{2} \approx 1.414 .
$$

The reciprocal $1 / K_{4}$ is a kind of capacity-it is the largest number so that every arc of that length lies in a drape of unit length. From (5) we see that $1 / K_{4} \geq 0.7070$.

\section{A Limit Theorem}

In the study of convexity for compact sets the Hausdorff metric is appropriate, but for arc covering problems one must also take the length of the arc into account.

To set our notation, we write $\operatorname{cl}(\mathcal{A}), \operatorname{int}(\mathcal{A})$, and $\partial \mathcal{A}=\operatorname{cl}(\mathcal{A}) \backslash \operatorname{int}(\mathcal{A})$ for the closure, interior, and topological boundary of the set $\mathcal{A}$. Let $D_{\varepsilon}(X)$ be the open disk of radius $\varepsilon$ with center $X$, and if $\mathcal{S}$ is a non-empty subset of $\mathbb{R}^{2}$ let

$$
\mathcal{S}_{\varepsilon}=\bigcup_{X \in S} D_{\varepsilon}(X)
$$

be the $\varepsilon$-neighborhood of $\mathcal{S}$. Let

$$
\operatorname{dist}(\mathcal{A}, \mathcal{B})=\operatorname{glb}\{A B: A \in \mathcal{A} \text { and } B \in \mathcal{B}\}
$$

be the Euclidean distance between the two sets $\mathcal{A}$ and $\mathcal{B}$, and let

$$
\mathfrak{h}(\mathcal{A}, \mathcal{B})=\operatorname{glb}\left\{r \geq 0: \mathcal{A} \subseteq \mathcal{B}_{r} \text { and } \mathcal{B} \subseteq \mathcal{A}_{r}\right\}
$$

be the Hausdorff distance between the two bounded sets $\mathcal{A}$ and $\mathcal{B}$. (For an investigation of the properties of this metric and a proof of the Blaschke Selection Theorem see [2, p. 133], [7, pp. 59-67], or [13, pp. 233-235]). 
If $\left\langle\gamma_{n}\right\rangle_{n=1}^{\infty}$ is a sequence of arcs $\gamma_{n}$ of length $L_{n}$ and if $\gamma$ is an arc of length $L$ so that $\mathfrak{h}\left(\left\{\gamma_{n}\right\},\{\gamma\}\right) \longrightarrow 0$, then

$$
\limsup _{n \rightarrow \infty} L_{n} \geq L .
$$

When the lengths also converge, we call the convergence "normal."

Definition. A sequence $\left\langle\gamma_{n}\right\rangle_{n=1}^{\infty}$ of arcs $\gamma_{n}$ with length $L_{n}$ converges normally to an arc $\gamma$ of length $L$, which we write $\gamma_{n} \rightrightarrows \gamma$, if both $h\left(\left\{\gamma_{n}\right\},\{\gamma\}\right) \longrightarrow 0$ and $L_{n} \longrightarrow L$.

It is clear and easily proved that if $\gamma_{n} \rightrightarrows \gamma$ and if $\mu$ is any rigid motion in the plane, then

$$
\mu \circ \gamma_{n} \rightrightarrows \mu \circ \gamma,
$$

so that arcs and their limits can be moved around in the plane at will.

Theorem 8. If $\gamma_{n}$ is a drapeable arc for each positive integer $n$ and if $\left\langle\gamma_{n}\right\rangle_{n=1}^{\infty}$ converges normally to an arc $\gamma$, then $\gamma$ is also a (possibly degenerate) drapeable arc.

Proof. For each $n$, let $\delta_{n}$ be a drape of length at most $l\left(\gamma_{n}\right)$ whose closed convex hull $\mathcal{H}_{n}$ covers $\left\{\gamma_{n}\right\}$. It entails no loss of generality to suppose that each $\delta_{n}$ lies in the upper half-plane with its initial point at the origin and its terminal point on the positive $x$-axis; and we may suppose further that $\left\{\gamma_{n}\right\} \subseteq \mathcal{H}_{n}$. The sequence $\left\langle\delta_{n}(1)\right\rangle$ of terminal points on the positive $x$-axis is bounded, so there is a subsequence (which we again denote $\left\langle\delta_{n}\right\rangle$ ) so that $\delta_{n}(1) \longrightarrow d$. Then the sequence $\left\langle\mathcal{H}_{n}\right\rangle$ is uniformly bounded, and according to the Blaschke Selection Theorem it has a subsequence (which again we denote by $\left\langle\mathcal{H}_{n}\right\rangle$ ) that converges to a compact, convex set $\mathcal{H}$, which clearly contains $\{\gamma\}$. The boundary arcs $\partial \mathcal{H}_{n}$, which have the form $\delta_{n} \oplus\left[0, \delta_{n}(1)\right]$, converge to the boundary arc $\partial \mathcal{H}$, and the line segment $\left[0, \delta_{n}(1)\right]$ converges to the line segment $[0, d]$. It follows that the drapes $\delta_{n}$ converge to a convex $\operatorname{arc} \delta$ whose convex hull is $\mathcal{H}$, and from

$$
l\left(\gamma_{n}\right) \geq l\left(\delta_{n}\right)=l\left(\partial \mathcal{H}_{n}\right)-\delta_{n}(1)
$$

we conclude that $l(\gamma) \geq l(\partial \mathcal{H})-d=l(\delta)$. So $\delta$ is a convex arc of length at most $l(\gamma)$ whose closed convex hull $\mathcal{H}$ contains $\gamma$.

\section{Wrapped Arcs}

A unit arc $\gamma$ that wraps around its endpoint segment should be drapeable, because its "outer boundary," the boundary of the unbounded component of the complement of its trace $\{\gamma\}$, which is formed from non-overlapping subarcs of $\gamma$, should-apart from outcroppings - be a closed curve of total length not larger than one, and consequently its convex hull surrounds $\gamma$ and has length at most one. This straightforward intuition turns out to be difficult to make precise. We attack this situation in a quite different way, using the language of integral geometry and the Cauchy-Crofton formula (see [6] or [19]), and we find a stronger result. 


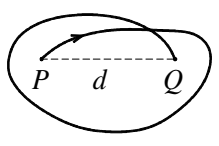

(a)

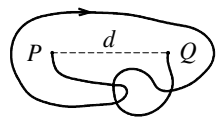

(b)

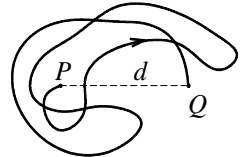

(c)

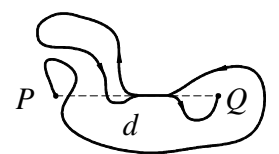

(d)

Fig. 10. Wrapped arcs.

Let $\mathcal{H}$ be the closed convex hull of the trace $\{\gamma\}$ of $\gamma$, and let $\partial \mathcal{H}$ be its boundary (which we regard here as a parameterized arc of length $l(\partial \mathcal{H})$ ). For each line $\ell$ let

$$
n_{\gamma}(\ell)=\#\{t \in[0,1]: \gamma(t) \in \ell\},
$$

which we take to lie in $\mathbb{N} \cup\{0, \infty\}$.

Definition. An arc $\gamma$ from $P$ to $Q \neq P$ is wrapped if $n_{\gamma}(\ell) \geq 2$ for every line $\ell$ that meets the endpoint segment $P Q$ (Fig. 10).

Recall the definition of geometric measure [6]: write each line $\ell$ in normal form

$$
x \cos \theta+y \sin \theta-p=0,
$$

where $p \geq 0$ and $0 \leq \theta<2 \pi$, and let $\ell^{*}$ be the point $(p, \theta)$ in the semi-infinite strip

$$
\mathbb{L}=\{(u, v): u \geq 0,0 \leq v<2 \pi\} .
$$

Then the geometric measure of the set $\Lambda$ is the Lebesgue measure of the set $\Lambda^{*} \subseteq \mathbb{L}$ of representative points $\ell^{*}$ for $\ell$ in $\Lambda$.

Lemma 9. The family $\Lambda$ of support lines of a compact, convex set $\mathcal{H}$ has geometric measure zero.

Proof. Each support line $\ell$ of $\mathcal{H}$ is parallel to at most one other support line of $\mathcal{H}$. So each horizontal line in $\mathbb{L}$ meets the set $\Lambda^{*}$ in at most two points, and the claim follows.

Theorem 10. Every wrapped arc $\gamma$ is drapeable.

Proof. We deduce from the Cauchy-Crofton formula that the boundary $\partial \mathcal{H}$ of the closed convex hull $\mathcal{H}$ of $\{\gamma\}$ has length at most one. Indeed,

$$
\begin{aligned}
2 l(\partial \mathcal{H}) & =\int_{\ell \cap \partial \mathcal{H} \neq \emptyset} n_{\partial \mathcal{H}}(\ell) d \ell \\
& =\int_{\ell \cap \partial \mathcal{H} \neq \emptyset} 2 d \ell
\end{aligned}
$$




$$
\begin{aligned}
& =\int_{\ell \cap\{\gamma\} \neq \emptyset} 2 d \ell \\
& \leq \int_{\ell \cap\{\gamma\} \neq \emptyset} n_{\gamma}(\ell) d \ell=2 l(\gamma)=2 .
\end{aligned}
$$

Formula (6) is just the Cauchy-Crofton formula. Formula (7) follows, because almost every line that meets the boundary $\partial \mathcal{H}$ of the closed convex hull $\mathcal{H}$ does so in two distinct points. (The lines that meet $\partial \mathcal{H}$ in just one point or in a line segment are support lines of $\mathcal{H}$, and they lie in a set of geometric measure zero by the lemma.) No line $\ell$ that meets $\partial \mathcal{H}$ can be disjoint from $\{\gamma\}$ because $\{\gamma\}$ is connected, and every line $\ell$ that meets $\{\gamma\}$ surely meets $\partial \mathcal{H}$. So the integrals (7) and (8) are over precisely the same set of lines.

Finally, suppose $\ell$ is a line that meets $\{\gamma\}$ for which $n_{\gamma}(\ell)=1$, and let $H_{\ell}^{+}$and $H_{\ell}^{-}$ be the two opposite open half-planes with edge $\ell$. If $\{\gamma\}$ meets both $H_{\ell}^{+}$and $H_{\ell}^{-}$, then $P$ and $Q$ lie on opposite sides of $\ell$. Consequently $\ell$ meets the endpoint segment $P Q$, and it follows that $n_{\gamma}(\ell) \geq 2$ because $\gamma$ is wrapped, a contradiction. So $\{\gamma\}$ lies entirely to one side or the other of $\ell$, which consequently is a support line of $\mathcal{H}$ and hence lies in a set of geometric measure zero by Lemma 9. Thus (9) follows. The last assertion is the Cauchy-Crofton formula again.

This theorem has an important consequence. If the endpoints of a unit arc $\gamma$ can be joined by an arc that is surrounded by $\gamma$, then $\gamma$ is drapeable. To be more precise, we define the core of $\gamma$ (Fig. 11).

Definition. Let $\gamma$ be an arc and let $C_{\infty}$ be the unbounded open connected component of the open set $\mathbb{R}^{2} \backslash\{\gamma\}$. We call the open set $\mathfrak{C}(\gamma)=\mathbb{R}^{2} \backslash \operatorname{cl}\left(C_{\infty}\right)$ the core of $\gamma$.

Corollary 11. Let $\gamma$ be a unit arc with distinct endpoints $P$ and $Q$. If there is an arc $\alpha$ from $P$ to $Q$ that apart from its endpoints lies in the core $\mathfrak{C}(\gamma)$ of $\gamma$, then $\gamma$ is drapeable.

Proof. We claim that $n_{\gamma}(\ell) \geq 2$ for every line $\ell$ that meets the endpoint segment $P Q$. If a line $\ell$ passes through an endpoint the inequality is obvious; and if $\ell$ meets $P Q$ at a point between the endpoints, then $\ell$ also meets $\{\alpha\}$ at a point of $\mathfrak{C}(\gamma)$, and that point splits $\ell$ into two opposite rays each of which meets $\{\gamma\}$; so again $n_{\gamma}(\ell) \geq 2$. Thus $\gamma$ is wrapped and consequently drapeable.

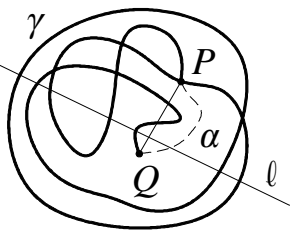

(a) Both endpoints in core.

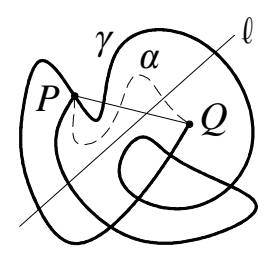

(b) One endpoint on boundary.

Fig. 11. Endpoints joined in the core. 
It is probably true that if $\gamma$ is wrapped, then $l(\gamma) \geq 3 d$, but we have been unable to deduce this from just the definition of wrapped. A slightly stronger hypothesis does suffice. Call an arc tightly wrapped if every ray $\overrightarrow{X Y}$ with endpoint $X$ on the endpoint segment $P Q$ meets the arc at a point different from the endpoint $X$. So, for example, the wrapped arcs pictured in Figs. 10(a),(b) are tightly wrapped, but those pictured in Figs. 10(c),(d) are not tightly wrapped.

Lemma 12 (Wetzel [25]). If an arc $\gamma$ is tightly wrapped (and, in particular, if the open endpoint segment $\stackrel{\circ}{P} \stackrel{\circ}{\mathrm{o}}$ the arc $\gamma$ lies in a bounded open connected component of $\left.\mathbb{R}^{2} \backslash\{\gamma\}\right)$, then $l(\gamma) \geq 3 d$.

Proof. We show that $n_{\gamma}(\ell) \geq 3$ for every line $\ell$ that meets the endpoint segment $P Q$, and then the conclusion follows from the Cauchy-Crofton formula. If $\ell$ passes through either $P$ or $Q$ (or both), this inequality is obvious, so suppose that $\ell$ meets $P Q$ at a point $R$ strictly between $P$ and $Q$. Let $X$ be the first point of $\gamma$ that lies on $\ell$. If $X=R$, then each of the two opposite rays formed on $\ell$ by the point $R$ contains a point of $\gamma$, so $n_{\gamma}(\ell) \geq 3$. Otherwise there is a point $Y$ beyond $X$ along $\gamma$ that lies on the ray opposite ray $R X$. If the subarc of $\gamma$ from $Y$ to $Q$ meets $\ell$ again, then $n_{\gamma}(\ell) \geq 3$. Otherwise the ray $R Y$ is a positive distance away from the subarc $\gamma_{P}$ from $P$ to $X$, and the ray $R X$ is a positive distance away from the subarc $\gamma_{Q}$ from $Y$ to $Q$, and so there is a line $m$ through $R$ that separates $\gamma_{P}$ and $\gamma_{Q}$ (see Fig. 12). By assumption, there are points $U$ and $V$ on $\gamma$ on opposite sides of $R$ along $m$, and those two points lie on opposite sides of $\ell$. Since $\gamma$ joins $U$ and $V$, it must cross $\ell$ again, and $n_{\gamma}(\ell) \geq 3$, as claimed. Now, according to the Cauchy-Crofton formula,

$$
2 l(\gamma)=\int_{\ell \cap \gamma \neq \emptyset} n_{\gamma}(\ell) d \ell \geq \int_{\ell \cap P Q \neq \emptyset} n_{\gamma}(\ell) d \ell \geq 3 \int_{\ell \cap P Q \neq \emptyset} d \ell=3(2 P Q),
$$

and the result follows.

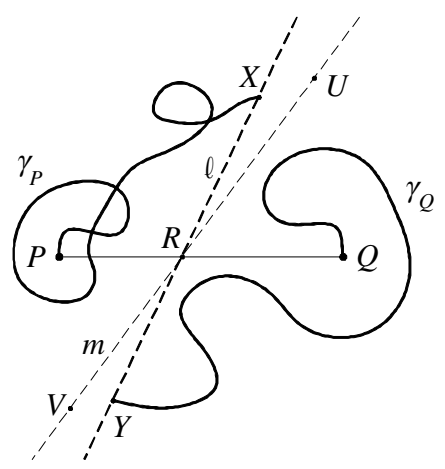

Fig. 12. The proof that $n_{\gamma}(\ell) \geq 3$. 


\section{Simple Approximation}

The fact that every arc can be suitably approximated by a simple arc really belongs to nineteenth century classical analysis. The informal claim that "covering simple arcs is enough" is mentioned without proof or attribution by Norwood and Poole [17, p. 410], and they assert that the proof is "straightforward point-set topology." Although we have not found a careful statement or detailed proof in the literature, we agree that the arguments are straightforward, but the details are a bit daunting. In this section we sketch an argument that "covering simple polygonal arcs" is enough.

Call two polygonal arcs "nearby" if their lengths are close and their traces are close in the Hausdorff metric. The argument requires moving the vertices of a polygonal arc to form a nearby polygonal arc that is, in some sense, more nearly simple. These moves rely on the following preliminary lemma.

Lemma 13. Let $\varpi=\left\langle V_{0} V_{1} V_{2} \cdots V_{n}\right\rangle$ be a polygonal arc, and suppose that $\varepsilon>0$ is given. If each of the vertices $V_{0}^{\prime}, V_{1}^{\prime}, V_{2}^{\prime}, \ldots, V_{n}^{\prime}$ of a polygonal path $\varpi^{\prime}=\left\langle V_{0}^{\prime} V_{1}^{\prime} V_{2}^{\prime} \cdots V_{n}^{\prime}\right\rangle$ lies within $\varepsilon /(3 n)$ of the corresponding vertex of $\varpi$, then $\left|l\left(\varpi^{\prime}\right)-l(\varpi)\right|<\varepsilon$ and $\mathfrak{h}\left(\varpi^{\prime}, \varpi\right)<\varepsilon$.

Proof. Let $\delta=\varepsilon /(3 n)$, and suppose that $V_{i}^{\prime} V_{i}<\delta$ for each $i$ (Fig. 13). Then

$$
V_{j-1} V_{j}-2 \delta<V_{j-1}^{\prime} V_{j}^{\prime}<V_{j-1} V_{j}+2 \delta
$$

for each $j=1,2, \ldots, n$, so that

$$
\left|l\left(\varpi^{\prime}\right)-l(\varpi)\right| \leq \sum_{j=1}^{n}\left|V_{j-1}^{\prime} V_{j}^{\prime}-V_{j-1} V_{j}\right|<2 n \delta<\varepsilon .
$$

Clearly $\left\{\varpi^{\prime}\right\} \subseteq\{\varpi\}_{\delta} \subseteq\{\varpi\}_{\varepsilon}$ (Fig. 13), and if $X$ is a point of $\{\varpi\}$ there is a point $X^{\prime}$ of $\left\{\varpi^{\prime}\right\}$ with $X^{\prime} X<\delta$, and so $\{\varpi\} \subseteq\left\{\varpi^{\prime}\right\}_{\delta} \subseteq\left\{\varpi^{\prime}\right\}_{\varepsilon}$. Hence $\mathfrak{h}\left(\{\varpi\},\left\{\varpi^{\prime}\right\}\right)<\varepsilon$.

Here is the main result.

Theorem 14 (The Simple Approximation Theorem). Let $\gamma$ be an arc of length $L>0$ having distinct endpoints $P$ and $Q$. For each $\varepsilon>0$ there is a simple polygonal path $\pi$ from $P$ to $Q$ such that $L-\varepsilon<l(\pi) \leq L$ and $\mathfrak{h}(\{\pi\},\{\gamma\})<\varepsilon$.

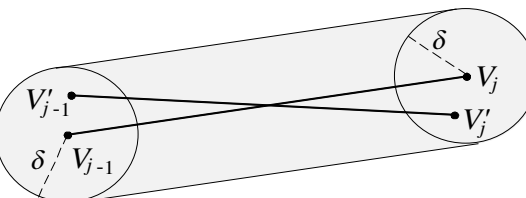

Fig. 13. Nearby vertices. 


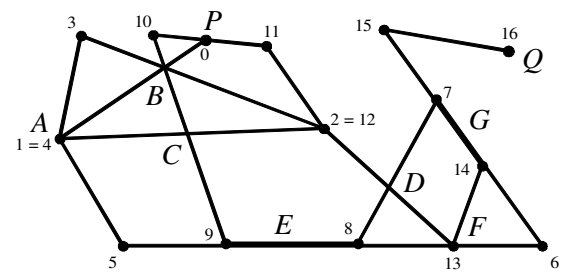

(a) A degenerate polygonal arc.

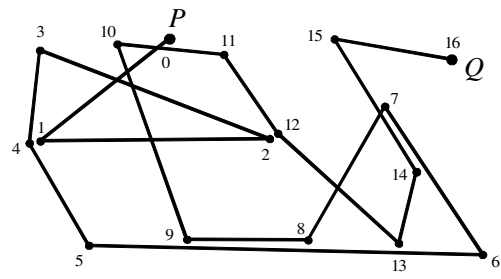

(b) A nearby tempered arc.

Fig. 14. A non-simple polygonal arc.

Proof. We content ourselves with a sketch of the argument. The cases in which $\gamma$ is a line segment of length $L$ being trivial, we assume that $P Q<L$. Because $\gamma$ is rectifiable there is a partition $\mathcal{P}=\left\langle t_{0}, t_{1}, t_{2}, \ldots, t_{n}\right\rangle$ of the parameter interval $[0,1]$ so that the length $l\left(\varpi_{0}\right)$ of the associated polygonal path

$$
\varpi_{0}=\left\langle\gamma\left(t_{0}\right) \gamma\left(t_{1}\right) \gamma\left(t_{2}\right) \cdots \gamma\left(t_{n}\right)\right\rangle
$$

lies in the range $L-\varepsilon<l\left(\varpi_{0}\right) \leq L$, and the length $l\left(\gamma_{i}\right)$ of each subarc $\gamma_{i}$ from $\gamma\left(t_{i-1}\right)$ to $\gamma\left(t_{i}\right)$ of $\varpi_{0}$ is less than $\varepsilon$. Then $\{\gamma\} \subset\left\{\varpi_{0}\right\}_{\varepsilon}$ and $\left\{\varpi_{0}\right\} \subset\{\gamma\}_{\varepsilon}$, so that $\mathfrak{h}\left(\{\gamma\},\left\{\varpi_{0}\right\}\right)<\varepsilon$.

Take $\delta$ so that $0<\delta<\varepsilon-\left(l(\gamma)-l\left(\varpi_{0}\right)\right)$. A polygonal arc $\varpi_{0}$, as an assemblage of segments, can fail to be simple in only a few ways (Fig. 14(a)): two or more vertices might coincide $(A)$; a vertex might lie on a non-adjacent edge $(F)$; different edges might have a line segment in common $(E, G)$; different edges might meet at a point not a vertex $(B, C, D)$. A point is a node if it lies on two non-overlapping, non-adjacent, intersecting edges of $\varpi_{0}$, and a node is ordinary if it lies on exactly two such edges. By making small adjustments in the positions of the vertices (Fig. 14(b)), we can find a polygonal arc $\varpi$ whose only degeneracies are ordinary nodes that lies near $\varpi_{0}$ in the sense that both $\mathfrak{h}\left(\{\varpi\},\left\{\varpi_{0}\right\}\right)<\delta$ and $\left|l(\varpi)-l\left(\varpi_{0}\right)\right|<\delta$. Then $\mathfrak{h}(\{\varpi\},\{\gamma\})<2 \varepsilon$ and $l(\gamma)-\varepsilon<l(\varpi) \leq l(\gamma)$.

It remains to eliminate the ordinary nodes and form a simple polygonal arc. Incorporate each node into the arc as a new vertex, and suppose the resulting polygonal arc is

$$
\pi_{0}=\left\langle P V_{1} V_{2} \cdots V_{N-1} Q\right\rangle .
$$

Suppose inductively that $i \geq 1$ is the smallest index so that the vertex $V_{i}$ is an ordinary node. There is a first index $j>i$ so that $V_{j}=V_{i}$. Then at $V_{i}$ the subarcs $\left\langle V_{i-1} V_{i} V_{i+1}\right\rangle$ and $\left\langle V_{j-1} V_{j} V_{j+1}\right\rangle$ cross or do not cross. Do nothing when they do not cross; but when they cross, replace the under-bracketed subarc

$$
\langle P \cdots V_{i-1} V_{i} \underbrace{V_{i+1} \cdots V_{j-1}} V_{j} V_{j+1} \cdots Q\rangle
$$

by its reversal, to give

$$
\langle P \cdots V_{i-1} V_{i} \underbrace{V_{j-1} V_{j-2} \cdots V_{i+1}} V_{j} V_{j+1} \cdots Q\rangle .
$$




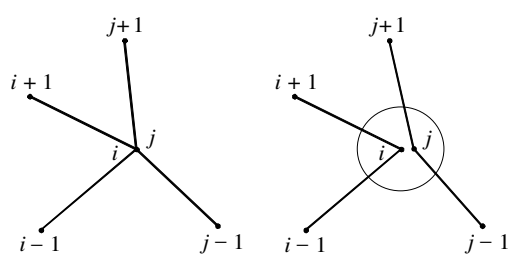

Fig. 15. Removing the node $V_{i}=V_{j}$.

In the resulting polygonal arc, the vertex $V_{i}$ is still incident to the same four edges, but the altered arc does not cross itself there. Now $V_{i}$ and $V_{j}$ can be separated a little (Fig. 15) to eliminate the node $V_{i}=V_{j}$ completely. Iterating this maneuver, we generate a simple polygonal arc $\pi$ that lies as close as desired to $\pi_{0}$ and consequently as close as desired to $\gamma$. This completes the sketch of the argument.

\section{Unilateral Arcs}

Let $\gamma$ be a unit arc with distinct endpoints $P$ and $Q$, and suppose that $\{\gamma\}$ meets the endpoint segment $P Q$ only at the endpoints. If $\gamma$ does not meet its open endpoint segment $\stackrel{P}{Q}$, then $\stackrel{\circ}{P} Q$ lies in a well-defined connected component $\mathcal{C}$ of $\mathbb{R}^{2} \backslash\{\gamma\}$. When $\mathcal{C}$ is a bounded component, $P Q$ defines an arc in the core $\mathfrak{C}(\gamma)$ of $\gamma$ that connects the endpoints of $\gamma$, and it follows from Corollary 11 that $\gamma$ is drapeable. In this section we prove that this same conclusion follows when $\stackrel{P}{P} \stackrel{\circ}{ }$ lies in the unbounded open connected component $\mathcal{C}_{\infty}$ of the complement of $\mathbb{R}^{2} \backslash\{\gamma\}$.

Noting that when $\gamma$ lies entirely to one side of the line through its endpoints, the drapeability follows from Corollary 2 , we call an arc $\gamma$ "unilateral" when $\stackrel{\circ}{P} \stackrel{\circ}{\text { lies in }} \mathcal{C}_{\infty}$.

Definition. An arc $\gamma$ is unilateral if its (open) endpoint segment $\stackrel{\circ}{P}$ lies in the unbounded open connected component $C_{\infty}$ of $\mathbb{R}^{2} \backslash\{\gamma\}$ (Fig. 16). A separator for a unilateral

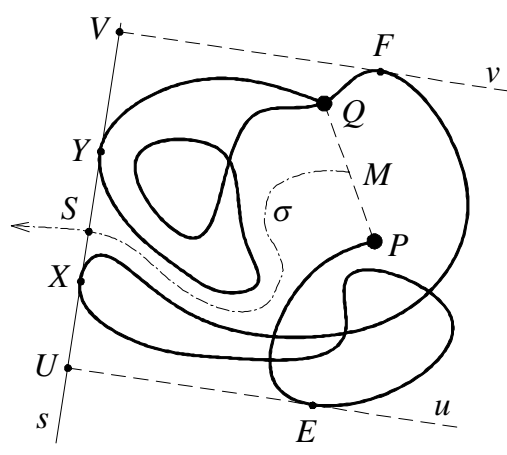

Fig. 16. A framed unilateral arc. 
arc $\gamma$ is a simple path $\sigma:[0, \infty) \longrightarrow C_{\infty}$ that joins the midpoint $M$ of the endpoint segment $P Q$ to the point at infinity (i.e., $\lim _{t \rightarrow \infty}|\sigma(t)|=\infty$ ).

Lemma 15. Suppose an arc $\gamma$ from $P$ to $Q \neq P$ is unilateral, and let $\mathcal{H}$ be the convex hull of its trace $\{\gamma\}$. Then $\gamma$ has a separator, and there exist a support line $s$ of $\mathcal{H}$ and three distinct points $X, S$, and $Y$ on $s$ so that (i) $S$ lies between $X$ and $Y$ on $s$; (ii) $S$ lies on $\sigma$, and $X$ and $Y$ lie on $\gamma$; (iii) no point between $X$ and $Y$ lies on $\gamma$; and (iv) $P, X, Y$, and $Q$ are in that order along $\gamma$ (Fig. 16).

Proof. The existence of a separator is clear. If the endpoint segment $P Q$ lies on the boundary of the convex hull $\mathcal{H}$ then we can take $S=M, X=P, Y=Q$, and $s$ the line $P Q$. Otherwise the midpoint $M$ of the segment $P Q$ lies in the interior of the hull $\mathcal{H}$. In this case there is a first point $S$ on the separator $\sigma$ that lies on the boundary $\partial \mathcal{H}$ of the hull, and that point $S$ lies in the interior of a well-defined shortest line segment in $\partial \mathcal{H}$. Take $s$ to be the line of this line segment, and label the endpoints of the segment $X$ and $Y$ in such a way that the points are in the order $P-X-Y-Q$ along $\gamma$.

We call the separator and the structure described in this lemma a frame for $\gamma$.

To show that an arbitrary unilateral unit arc is drapeable, we show first that simple unilateral unit arcs are drapeable and then approximate.

Theorem 16. Every simple unilateral arc $\gamma$ is drapeable.

Proof. Let $\gamma$ be a simple unilateral unit arc of length $L$ from $P$ to $Q \neq P$. The construction is pictured in Fig. 17. Let $\mathcal{H}$ be the closed convex hull of $\gamma$ and let $\sigma, s$, $S, X$, and $Y$ be a frame for $\gamma$ as described in Lemma 15. Let $U$ and $V$ be the points on the rays $S X$ and $S Y$, respectively, where the support lines $u$ and $v$ of $\mathcal{H}$ perpendicular to $s$ meet $s$, and let $E$ and $F$ be points where $u$ and $v$, respectively, touch $\{\gamma\}$. Then the points along $\gamma$ are in the order $P-X-E-F-Y-Q$ because $\gamma$ is simple. The four points $X, E, F$, and $Y$ partition $\gamma$ into five subarcs, $\gamma_{P}^{X}, \gamma_{X}^{E}, \gamma_{E}^{F}, \gamma_{F}^{Y}$, and $\gamma_{Y}^{Q}$ (some might degenerate to points). Let $\delta$ be the arc from $X$ to $Y$ on the boundary of the closed convex hull of the closed curve

$$
Y X \oplus \gamma_{X}^{E} \oplus \gamma_{E}^{F} \oplus \gamma_{F}^{Y}
$$

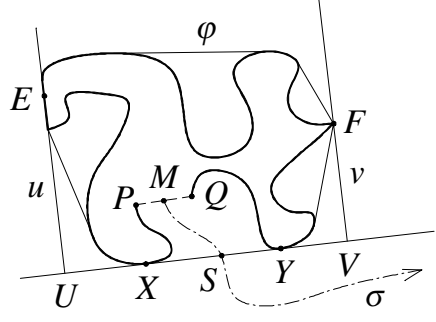

Fig. 17. A simple unilateral arc. 
Then

$$
l(\delta)+X Y \leq l\left(\gamma_{X}^{E}\right)+l\left(\gamma_{E}^{F}\right)+l\left(\gamma_{F}^{Y}\right)+X Y
$$

(because the boundary curve of the convex hull of a closed curve is no longer than the curve itself), and

$$
E U \leq l\left(\gamma_{X}^{E}\right) \leq l\left(\gamma_{X}^{E}\right)+l\left(\gamma_{P}^{X}\right)
$$

and

$$
F V \leq l\left(\gamma_{F}^{Y}\right) \leq l\left(\gamma_{F}^{Y}\right)+l\left(\gamma_{Y}^{Q}\right) .
$$

Let $\delta_{E}^{F}$ be the subarc of $\delta$ from $E$ to $F$. Then the convex arc

$$
\varphi=F V \oplus \delta_{E}^{F} \oplus U E
$$

is a drape whose closed convex hull contains that of $\{\gamma\}$, and $l(\varphi) \leq L=l(\gamma)$.

Theorem 17. Every unilateral arc is drapeable.

Proof. We content ourselves with a sketch of the proof. Let $\gamma$ be a unilateral arc of length $L$, endpoints $P$ and $Q$, and separator $\sigma$. Let $M$ be the midpoint of $P Q$, and for each sufficiently large positive integer $n$ let $X_{n}$ and $Y_{n}$ be the points on $P Q$ in the order $P-X_{n}-M-Y_{n}-Q$ so that $P X_{n}=Y_{n} Q=1 / n$. Let

$$
\mathcal{G}_{n}=\mathbb{R}^{2} \backslash\left(X_{n} Y_{n} \cup\{\sigma(t): t \geq 0\}\right) .
$$

Then $P$ and $Q$ lie in the open set $\mathcal{G}_{n}$, and for $n$ sufficiently large there is a simple polygonal arc $\varpi_{n}$ in $\mathcal{G}_{n}$ so that

$$
\begin{aligned}
\mathfrak{h}\left(\left\{\varpi_{n}\right\},\{\gamma\}\right) & <\frac{1}{n}, \\
L-\frac{1}{n} & <l\left(\varpi_{n}\right) \leq L,
\end{aligned}
$$

which we can choose so as to be disjoint from the entire open endpoint segment $\stackrel{P}{Q} Q$ by a minor modification of the argument sketched in the proof of Theorem 14. Then the polygonal arc

$$
\pi_{n}=Y_{n} Q \oplus \varpi_{n} \oplus P X_{n}
$$

is simple and unilateral and consequently drapeable (by Theorem 16), and the sequence $\left\langle\pi_{n}\right\rangle$ converges normally to $\gamma$. It follows from Theorem 8 that $\gamma$ is drapeable.

\section{A Concluding Remark}

We have shown that a non-drapeable unit arc $\gamma$ with endpoints $P$ and $Q$ must have $P Q>0.2220$, it must meet and cross its open endpoint segment $\stackrel{\circ}{P} \stackrel{\circ}{ }$, and the set of lines $\ell$ meeting the endpoint segment $P Q$ for which $n_{\gamma}(\ell)=1$ must have positive geometric measure. These conditions are far from strong enough to characterize nondrapeability. 


\section{Acknowledgments}

We acknowledge with pleasure many fruitful conversations with J. Ralph Alexander, Mary-Elizabeth Hamstrom, Kyung-won Hwang, and other participants in the Geometric Potpourri Seminar at the University of Illinois and with Songkiat Sumetkijakan at Chulalongkorn University. We express our gratitude to Faisal G. Mohamed at UIUC for some insightful computational work with Mathematica.

\section{References}

1. A. S. Besicovitch, On arcs that cannot be covered by an open equilateral triangle of side 1, Math. Gaz. 49 (1965), 286-288.

2. R. V. Benson, Euclidean Geometry and Convexity, McGraw-Hill, New York, 1966.

3. P. R. Coulton, Perhaps the 30 degree pie slice covers three ligament worms, Geometric Potpourri Seminar presentation at the University of Illinois, Urbana, IL, June 10, 2003.

4. H. S. M. Coxeter and S. L. Greitzer, Geometry Revisited, Random House, New York, 1967.

5. H. T. Croft, K. J. Falconer, and R. K. Guy, Unsolved Problems in Geometry, Springer-Verlag, New York, 1991.

6. M. P. do Carmo, Differential Geometry of Curves and Surfaces, Prentice-Hall, Englewood Cliffs, NJ, 1976.

7. H. G. Eggleston, Convexity, Cambridge University Press, Cambridge, 1958.

8. S. R. Finch, Mathematical Constants, Cambridge University Press, Cambridge, 2003.

9. S. R. Finch and J. E. Wetzel, Lost in a forest, Amer. Math. Monthly 111 (2004), 645-654.

10. J. Gerriets and G. Poole, Convex regions which cover arcs of constant length, Amer. Math. Monthly $\mathbf{8 1}$ (1974), 36-41.

11. R. Graham, Problem 41, Proceedings, 1963 Number Theory Conference, University of Colorado, Boulder, CO, 1963.

12. J. A. Johnson, G. D. Poole, and J. E. Wetzel, A small cover for convex unit arcs, Discrete Comput. Geom. 32 (2004), 141-147.

13. P. J. Kelly and M. L. Weiss, Geometry and Convexity, Wiley, New York, 1979.

14. J. H. McKay, The William Lowell Putnam Mathematical Competition, Amer. Math. Monthly 77 (1970), 721-728.

15. L. Moser, Poorly formulated unsolved problems in combinatorial geometry, Mimeographed, undated (but about 1966).

16. W. O. J. Moser, Problems, problems, problems, Discrete Appl. Math. 31 (1991), 201-225.

17. R. Norwood and G. Poole, An improved upper bound for Leo Moser's worm problem, Discrete Comput. Geom. 29 (2003), 409-417.

18. J. Pál, Über ein elementares Variationesproblem, Math. Fys. Medd. Danske Vid. Selsk. 3 (1920), No. 2, $35 \mathrm{pp}$.

19. L. A. Santaló, Integral Geometry and Geometric Probability, vol. 1 of Encyclopedia of Mathematics and its Applications, G.-C. Rota, ed., Addison-Wesley, Reading, MA, 1976.

20. J. Schaer, An "obvious" but useful theorem about closed curves, Math. Mag. 45 (1972), 154-155.

21. J. Schaer and J. E. Wetzel, Boxes for curves of constant length, Israel J. Math. 12 (1972), 257-265.

22. W. Wichiramala, A smaller cover for unit convex arcs, Preprint.

23. J. E. Wetzel, Sectorial covers for curves of constant length, Canad. Math. Bull. 16 (1973), 367-375

24. J. E. Wetzel, Fits and covers, Math. Mag. 76 (2003), 349-363, 398.

25. J. E. Wetzel, Problem 11064, Amer. Math. Monthly 111 (2004), 165.

26. J. E. Wetzel, The classical worm problem-a status report, Geombinatorics, to appear.

Received January 31, 2004, and in revised form May 13, 2005. Online publication August 23, 2005. 